\title{
Pyramid Projection - Validation of a New Method of Skin Defect Measurement
}

\author{
J. RŮŽIČKA ${ }^{1}$, P. NOVÝ ${ }^{2}$, F. VÁVRA ${ }^{2}$, L. BOLEK $^{1}$, J. BENES $\check{S}^{1}$ \\ ${ }^{1}$ Department of Biophysics, Medical Faculty of Charles University, Plzeñ, ${ }^{2}$ University of West \\ Bohemia, Plzeň, Czech Republic
}

Received May 23, 2007

Accepted May 29, 2007

On-line available May 31, 2007

\begin{abstract}
Summary
This paper presents a new method for the determination of the volume, surface area and depth of skin defects. The method is based on the description of a spatial defect using a pyramid (made, for example, from injection needles), which is placed over the defect. The projection of the pyramid on to the defect is photographed using a digital camera and subsequently compared with the projection of the same pyramid on to a sheet of grid paper. The defect is mathematically reconstructed on a computer, and an optimal body shape describing the defect is found, using a number of simplifications and assumptions. The method was then validated using a plaster mold of a real foot with 19 defects simulating real wounds. These plaster wounds were molded using alginate hydrocolloid, and the volume, surface area and depth were measured and compared with the results of the pyramid projection by means of regression analysis.

This method correlates in all variables with correlation coefficients higher than 0.9. It can be concluded that the projection pyramid method correlates well with the reference mold method and can be used with good results for a whole range of variables.
\end{abstract}

Key words

Wound $\bullet$ Ulcer $\bullet$ Defect measurement $\bullet$ Projection

\section{Introduction}

The most frequently used tools for measuring wound dimension are rulers or ruler-like devices making it possible to quantify the size, e.g. length $\mathrm{x}$ width or size A $x$ size B. These are complicated by certain problems, such as irregular wound shape, changing shape during healing, or undermining, and because of these factors more complex methods have been invented. Wound depth can be measured by a single gauge. All three dimensions should be measured by different mechanical devices such as the Kundin instrument (Wysocki 1996).

Wound area is also quite frequently measured. The simplest method is manual tracing on a cellophane film or plastic bag with successive evaluation - either manually by square summing on a superposed paper grid, or semi-automatically by a PC. This computerized planimetry is considered a gold standard, in spite of inaccuracies caused by double investigators (first: manual tracing by pen on film; second: outlining with a mouse on a PC). Photogrammetry is based on the known actual focal length of a camera lens capturing a sharp 
photograph of a wound. The defect area is determined by weighing of photograph paper or, in more modern practice, by computer planimetry. A fully automated version is a variant known as "gray level threshold technique" which evaluates a gray scale pixel distribution curve (Wysocki 1996).

None of these techniques can provide substantial information about volume. If a deep defect begins to heal from its base, the rate of healing can be rapid while the area can remain constant (or even slightly enlarged). Hence, the investigators looked for methods that could also offer information on volume. The most widespread volume measurement method is the mold technique, which uses dental materials - alginate hydrocolloids or silicon rubber (rarely). The volume of the mold is then measured by the Archimedes principle in a cylinder or else the mold is weighed in the case of known specific gravity - the gravimetry method (Stotts et al. 1996). One variant of this technique is measurement of volume by filling with saline (this is less exact). The main disadvantage of these methods is the epidemiological risks of their invasive character. They are not able to preserve wound shape for an extended period and they require staff experience.

Subsequently other, more sophisticated, volumetric methods were invented. Perhaps the most widely publicized are structure-light techniques, generally based on the triangulation principle. The MAVIS system (Plassmann et al. 1998) illuminates the wound from an exact angle by a set of parallel strips, and a CCD camera screens the image. Strips are color-coded to prevent image imperfections; from the strip distortions on the wound surface a computer calculates semiautomatically the parameters of the wound - volume, area, depth and circumference. (The wound border has to be inserted by mouse tracing). A variant of this method illuminates the wound from an exact angle by a net of laser beams forming a network of points on the surface of the wound. The computer analyzes the projection/distortion of the point images on the wound surface (Platete et al. 1996).

Another possibility is to scan mechanically the wound surface with a single (laser) beam and to measure the volume of the defect by tracing the reflection of the beam on a sensor.

All the aforementioned sophisticated methods are made more difficult by the fact that the screening takes some time and so motion of the patient/tissue causes inaccuracies in the results. The methods are not fully automated - the investigator has to trace the border of the defect so this is another source of inaccuracy.

Stereophotogrammetry, and its computerized version stereophotography, are based on the evaluation of an optical net projected on to a wound surface and captured by two (or more) cameras. These are considered to be the gold standard in volumetric techniques (Plassmann et al. 1994). Some systems use the holographic principle to measure the volume of a defect.

All these semi- or fully automated methods require a pool of expensive devices. The requirement for exact work with $\mathrm{CCD}$ cameras and projectors, the needs of strict calibration, etc., on restrict the use of these methods in routine clinical practice and confine them to the scientific field.

As far as the authors are aware there exists no simple, cheap, device-free and zero contact method for measuring the volume of a defect. The aim of this paper is to introduce and validate such a method requiring the following tools: an ordinary digital camera, a PC, two beads and six injection needles forming a pyramid.

\section{Methods}

\section{Basic principle and restrictions}

The method presented here utilizes the comparison of a pyramid projection (made, for example, from hypodermic needles) on to the surface of the defect and on a sheet of grid paper. To measure the defect volume/area according to this method it is necessary to construct a pyramid, to place it on the grid paper and to take a digital photograph. Then the pyramid is moved to the surface of the defect and a set of digital photos is taken from all three sides of the pyramid (see Fig. 1).

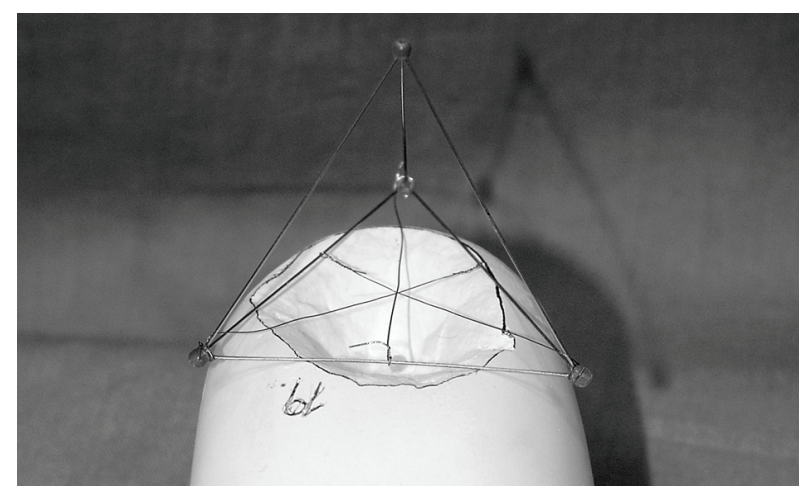

Fig. 1. Projection of the pyramid on to the defect 
These four photos clearly provide all the information necessary for 3-D description of the defect - i.e. volume calculation. Projection of the pyramid as a photo defines the real angles and dimensions during the shooting of the pictures. Software, including the appropriate mathematical techniques, has been developed to make it possible to calculate the volume/area of a defect from projections - with a number of restrictions that are described below.

The method presumes the following simplifications:

- A sufficient definition of the wound is a spatial (not planar) indication of (a) surface border of the defect and (b) its deepest point (or abscissa).

- A wound tends to minimize its surface tension like a soap bubble. Its ideal shape of minimal surface tension (e.g. a ball) is distorted by spatial definitions of its border and its deepest point

- The minimum possible volume is the mathematical space of a sphere cap; the maximal possible volume is the mathematical space of a cylinder. There exist other mathematical bodies - paraboloid, spire, cone and combinations of these.

The software utilizes the boundary of the defect and the deepest point of all three photos as a sufficient description of the defect. This information is entered manually into the computer - the defect boundary is traced by the mouse and the deepest point is selected. The problem of this method is the mathematical description of this process, which has to be a) sufficiently exact and b) resistant to human error during the manual input.

The exact description of the method presented here can be divided into the following steps:

1. By means of observation define uniquely the border of the defect

2. Insert a sterile bead at the deepest point of the defect. If it is not a point but an area that is deepest, insert two beads marking abscissa of longest diameter of this area. This is necessary for further recognition of the deepest point/area.

3. Place the pyramid over the defect. Observing the wound perpendicularly, set the tetrahedron to have its apex superposing with one or more beads

4. Make 3 digital photos of this from all sides of the pyramid.

5. If it has not been done before, make a calibration photo of the pyramid on grid paper (such as ECG paper)

6. Import the photos into the computer and process the data with the software. The input data are: all 4 pyramid apexes, the trace of the defect border and its deepest point (position of bead/beads) from all 3 projections. The software calculates the defect area, its depth, surface and volume.

The process of spatial description of the defect can be broken down into the following steps:

1. Simplification of the problem - the defect is sufficiently described by the boundary and the deepest point/abscissa

2. Reconstruction of 3-D information (defect boundary and deepest point) from a 2-D photo

3. Searching for the optimum body shape to describe the defect

In what follows the most important tasks and problems involved in these steps will be explained. Because of the extent of the precise mathematical description, the interpretation of the methodology is restricted simply to a verbal description, and the precise mathematical mechanism is given in the references (internet).

\section{Reconstruction of 3-D information from 2-D picture}

To take a photo means in fact to transform a 3-D space into a 2-D picture. A reverse transformation is possible only with subsequent knowledge, such as knowledge of additional photos made from different and known angles, as is used in cartography in the creation of maps. This method is not used here.

The next possibility (used here) is photographing a known body (pyramid) combined with some additional knowledge: (a) the pyramid bottom lies in the defect plain, and (b) the defect's deepest point lies below the apex of the pyramid. The spatial reconstruction means comparing the known information about the pyramid (size, geometrical laws) with its 2-D image on grid paper - i.e. to define the projection equations.

The projection equations can in principle be of two types: parallel and perspective.

A perspective (vanishing) projection supposes projection of the spatial body into the projection plane from one single point - the point of the observer. A parallel (coplanar) projection supposes the transposition of the space body into the projection plane by contraction, translation and rotation. The observer is a plane, and points are projected by parallel lines. 
Table 1. Results of reference measurements (mold method) for the pyramid projection calculations (projection method)

\begin{tabular}{|c|c|c|c|c|c|c|}
\hline \multirow[b]{2}{*}{ Defect No. } & \multicolumn{3}{|c|}{ MOLD METHOD } & \multicolumn{3}{|c|}{ PROJECTION METHOD } \\
\hline & Volume $\left[\mathrm{cm}^{3}\right]$ & Surface $\left[\mathrm{cm}^{2}\right]$ & Depth $[\mathrm{cm}]$ & Volume $\left[\mathrm{cm}^{3}\right]$ & Surface $\left[\mathrm{cm}^{2}\right]$ & Depth $[\mathrm{cm}]$ \\
\hline 1 & 0.37 & 2.500 & 0.342 & 0.379 & 2.31 & 0.31 \\
\hline 2 & 0.55 & 2.625 & 0.278 & 0.389 & 2.90 & 0.26 \\
\hline 3 & 0.43 & 2.375 & 0.300 & 0.565 & 2.61 & 0.40 \\
\hline 4 & 1.54 & 5.500 & 0.556 & 1.422 & 5.84 & 0.46 \\
\hline 5 & 0.99 & 4.625 & 0.418 & 1.290 & 5.57 & 0.44 \\
\hline 6 & 1.20 & 5.125 & 0.610 & 1.443 & 4.69 & 0.57 \\
\hline 7 & 0.12 & 0.505 & 0.158 & 0.076 & 0.62 & 0.20 \\
\hline 8 & 0.11 & 0.220 & 0.092 & 0.028 & 0.32 & 0.16 \\
\hline 9 & 0.18 & 0.860 & 0.168 & 0.080 & 1.04 & 0.14 \\
\hline 10 & 0.97 & 3.375 & 0.737 & 1.170 & 3.61 & 0.60 \\
\hline 11 & 1.05 & 3.250 & 0.683 & 0.922 & 3.65 & 0.47 \\
\hline 12 & 1.24 & 4.125 & 0.548 & 1.172 & 4.56 & 0.49 \\
\hline 13 & 2.35 & 5.500 & 0.931 & 2.500 & 5.81 & 0.78 \\
\hline 14 & 1.87 & 5.000 & 0.899 & 2.280 & 5.49 & 0.75 \\
\hline 15 & 2.61 & 6.375 & 0.863 & 3.650 & 7.74 & 0.86 \\
\hline 16 & 1.56 & 5.500 & 0.801 & 1.710 & 5.39 & 0.60 \\
\hline 17 & 1.01 & 3.250 & 0.647 & 1.723 & 3.97 & 0.75 \\
\hline 18 & 1.39 & 6.750 & 0.260 & 1.068 & 7.04 & 0.30 \\
\hline 19 & 8.42 & 13.750 & 1.470 & 10.010 & 14.73 & 1.20 \\
\hline
\end{tabular}

Table 2. Results of regression analysis

\begin{tabular}{llll}
\hline $\mathbf{P}=\mathbf{k} \cdot \mathbf{R}+\mathbf{q}$ & Surface & Volume & Depth \\
\hline$K$ & 1.060 & 1.200 & 0.760 \\
$Q$ & 0.080 & -0.090 & 0.080 \\
$\begin{array}{l}\text { Pearson } \\
\text { coefficient }\end{array}$ & 0.993 & 0.992 & 0.964 \\
$\begin{array}{l}\text { Spearman } \\
\text { coefficient }\end{array}$ & 0.969 & 0.914 & 0.959 \\
\hline
\end{tabular}

The reality of digital photography is more similar to the perspective projection. Unfortunately after construction of a mathematical apparatus, it was found that this projection is exact but very sensitive to inaccuracy in the definition of the pyramid point. Even a small deviation in mouse click causes a high variation in the result.

For these implemental reasons a parallel projection was chosen. This is less exact but more robust compared to the perspective projection.
Searching for the optimum body to describe the defect

The optimum geometrical body matches the defect surface totally. Hence, this is not accessible by the principle chosen by this method; we are seeking for the body showing the greatest similarity. In what follows, we present a list of the presumptions and simplifications that were used in this step:

Geometric law

- Cavalier's principle: two bodies with the same base and sagitta have the same volume if the sections at the same distance from the base have the same surface area.

\section{Extremum properties}

- A circle has the minimum surface for a given section value.

- A sphere has the minimum surface area for a given volume value.

\section{Physiological presumption}

- A defect tends to minimize a perimeter for the given section value. The shape of the boundary curve is 

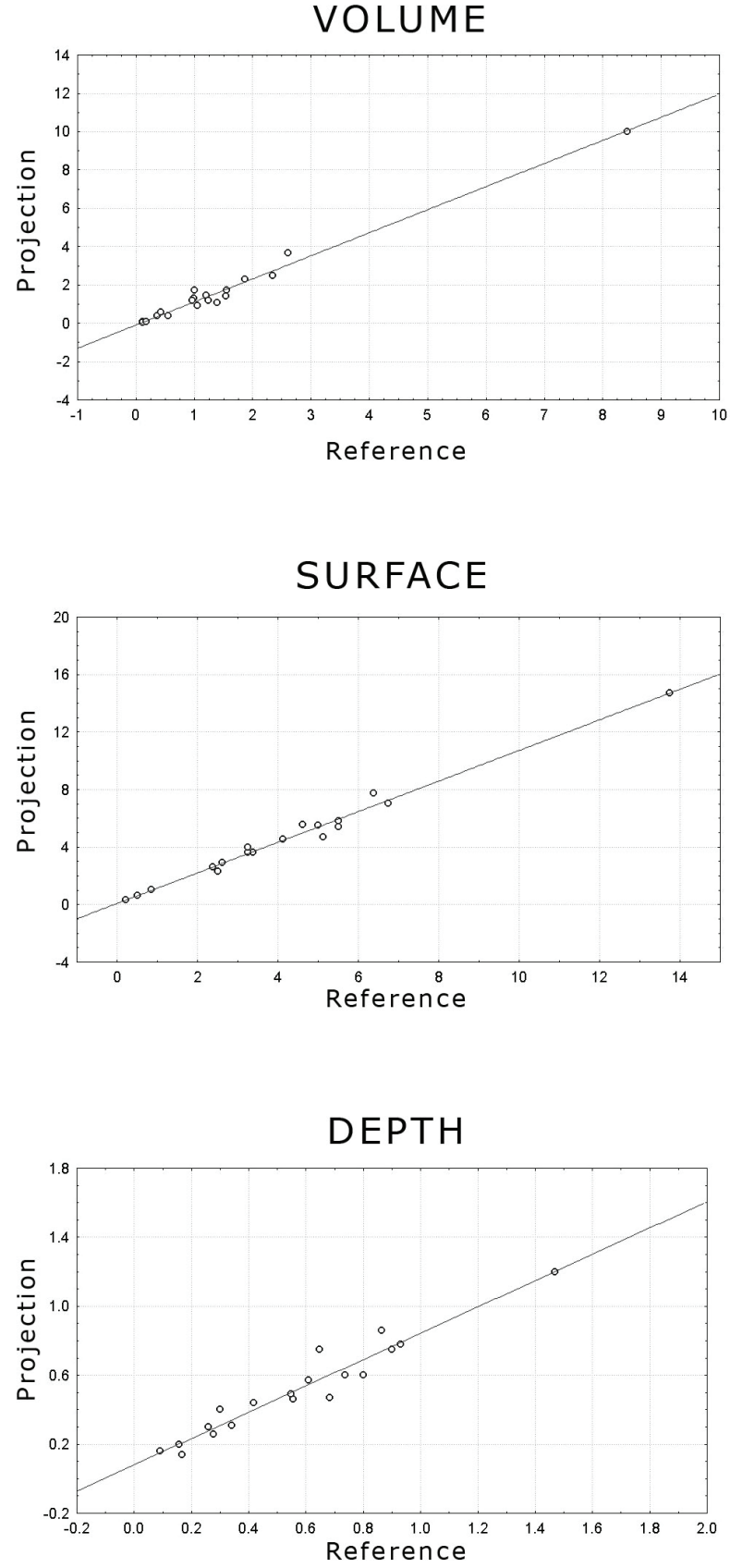

Fig. 2. Regression curves of variable volume, area and depth

influenced by binding to the surrounding tissue. Under the condition of no binding, the defect has a circular or elliptical shape.

In the process of programming the formula for the optimum geometrical body was established experimentally by first using 10 plaster molds (see below) which were tested by the following bodies: spire, sphere, cap and cylinder. Furthermore, the base of these bodies was calculated in two different ways: by using the perimeter and by using the surface (determined by numeric integration). The detailed mathematical mechanism that gives the best representation of the entity concerned is given in the references (internet).

\section{Method verification}

The described method exploits a set of simplifications and presumptions. Therefore, validation using a defect of real shape has to be done prior to use.

Eight plaster molds of a real foot were made and 19 defects were constructed in plaster simulating real wounds found in diabetic patients.

As reference - "gold standard" the following procedures were used:

- Area - the defect surface was evaluated by a grid method using a 1-mm grid paper.

- Volume - an alginate hydrocolloid (dental material) mold was made and the volume of the defect was measured by the mass of the mold (gravimetry) and by cylinder. Three molds of each defect were made to check reproducibility of the method.

- Depth - depth of the defect was measured with a caliper on each mold.

The described pyramid projection method was then checked on these foot molds. To provide exact pictures two pyramids of thin wire (edge size $60 \mathrm{~mm}$ and $15 \mathrm{~mm}$ ) were constructed instead of the needle pyramid, as illustrated in Fig. 1. The pyramid was chosen according to the best fit (i.e. best contact) with the defect surface.

Statistical analysis was performed using STATISTICA 98 software to prove the validity of the projection method explained here.

\section{Results}

The results are shown in Table 1 . The results of the reference measurements for the pyramid projection are displayed for the variable surface, volume and depth of the measured defect.

Sequentially regression curves were estimated and linear regression was used with good results. Pearson correlation coefficients and further Rank order Spearman correlation coefficients were calculated as parameters of the closeness of approach.

The results of regression analysis are shown in Table 2, containing the parameters of the general linear regression formula $P=k \cdot R+q$, where $P$ is the projection method variable and $R$ is the reference 
variable. Regression curves are shown in Fig. 2. Both Pearson and Spearman coefficients are of statistical significance $(p<0.001)$. Owing to the fact that no variable has a normal distribution the Spearman coefficient is more appropriate. From regression analysis, it can be concluded that the projection pyramid method correlates well with the reference mold method and can be used with good results in the whole value range of the variables being tested.

\section{Discussion}

A number of studies evaluate a different therapeutical approach using a presentation of quite simple terms such as "healed" or "improved", measured by the ruler method. As a general methodological characteristic of these studies, a lack of appropriate wound measurement/evaluation could be noted. Furthermore, routine clinical medicine does not possess a simple, cheap, accurate and reproducible method of wound evaluation.

This paper presents a method for measuring wound volume, depth and surface area using a basic digital camera, a PC, needles and grid paper. It supposes that wound shape is similar to mathematical bodies with respect to the Cavalier principle. Mathematical computations used in the software presume certain simplifications as described above. There may be problems in the measurement of defects with a very irregular shape, which do not fit with the mathematical bodies used in computations. Plaster molds were created in an attempt to cope with these irregularities but nevertheless in such cases the validation needs to be repeated with a range of different wounds.

Statistical analysis of the tested plaster models has yielded satisfactory results. It seems that the method is more accurate for larger defects: this is probably caused by the fact that a small pyramid does not fit well on a small defect and oscillates more, with the result that the small pyramid is relatively more crudely made in comparison with a larger one. The values of correlation coefficients and regression formulae show that the method is most precise for the determination of a surface that is almost equal to the reference molds. This is in accordance with the fact that the determination of surface is rendered more difficult by parallel projection error, in contrast to the determination of volume where the best body fit method leads to simplifications that cause inaccuracies.

As reference, an alginate mold was used. Sometimes it proved to be difficult to mold an exact foot shape in the alginate material. Another source of inaccuracy is the fact that alginate hydrocolloid changes its density over time (i.e. it dries out). As a result of this, the alginate mold method also cannot be considered to be perfectly exact.

Initially the alginate mold was made on real defects in diabetic patients. The movement of patients and the fact that the tissue of a defect is not firm causes a high degree of inaccuracy in the molding. Using a plaster foot was found to be better solution with defects, since this provided repeated molds without changes in the defect.

The time aspect was found to be very important. The alginate mold method is time consuming - it takes more than $15 \mathrm{~min}$ to prepare everything, to mix the alginate, to wait till the alginate solidifies, to measure the cast and so on. In contrast, to take three pictures with a digital camera takes less than one minute.

Further evaluation of this method is necessary. It would be beneficial to compare the method with some non-contact method, such as a structure-light technique (MAVIS or some other device described in the Introduction). Unfortunately, the authors of this paper do not possess any of these sophisticated devices. The software for the method presented here (including detailed mathematical derivations) is accessible on the internet and the authors are prepared to help all who are interested. An additional validation of the method could therefore be a goal for some independent workplace equipped with the mentioned reference device.

\section{Acknowledgements}

Grant support VZ MSMT CR 21620814.

\section{References}

Internet http://biofyzika.lfp.cuni.cz/pyramid/manus_phys_res.pdf

PLASSMANN P, JONES TD: MAVIS, a noninvasive instrument to measure area and volume of wounds. Med Eng Phys 20: 332-338, 1998. 
PLASSMANN P, MELHUISH JM, HARDING KG: Methods of measuring wound size - a comparative study, Wounds 6: 54-61, 1994.

PLATETE P, BULGRIN J, SHABANI M, SMITH D: A noninvasive, three-dimensional, diagnostic laser imaging system for accurate wound analysis, Physiol Meas 17: 71-79, 1996.

STOTTS N, SALAZAR M, TEVIS D, MCADOO E: Accuracy of alginate moulds for measuring Wound volumes when prepared and stored under varying conditions, Wounds 8: 158-164, 1996.

WYSOCKI A: Wound measurement. Int J Dermatol 35: 82-91, 1996.

\section{Corresponding author}

Jiří Růžička, Department of Biophysics, Faculty of Medicine, Charles University, Karlovarská 48, 30100 Plzeň, Czech Republic. E-mail: benes@dante.lfp.cuni.cz. 